\title{
Access Zone Luminance Prediction for a Road Tunnel under a Cloudless Sky
}

\author{
Wei He ${ }^{1,2}$ and Bo Liang ${ }^{1, *}$

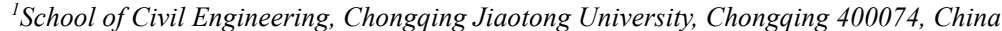 \\ ${ }^{2}$ Department of Traffic and Municipal Engineering, Sichuan College of Architectural Technology, Chengdu 610399, China
}

Received 3 August 2021; Accepted 29 October 2021

\begin{abstract}
Access zone luminance is affected by many factors, such as weather, test time, the location of the tunnel, the inclination of the portal, the orientation of the portal, and the colors of objects outside the tunnel. It is considerably difficult to predict. In order to predict access zone luminance under a cloudless sky accurately, the model for object surface luminance was proposed. The law about ratio of the normal luminance to the illuminance on the object surface was found through measurement. The influence of viewing angle on the luminance of a typical object outside the tunnel was analyzed. The predicted object surface luminance and access zone luminance were verified using the corresponding measured values, and the reasons for prediction error were analyzed. Results show that the daylight received by an object within a 20-degree field of view is approximately uniformly reflected in all directions. The ratio of luminance to illuminance on the surface of the object with a specific color can be supposed as constant when predicting access zone luminance. The proposed access zone luminance prediction method under a cloudless sky has good versatility, and the input parameters of the prediction method include all factors that affect access zone luminance. The relative root-meansquare error and relative mean bias error of the predicted access zone luminance in the case are $11.6 \%$ and $11.4 \%$, respectively. This study provides a reference for intelligent dimming of highway tunnel lighting.
\end{abstract}

Keywords: Illuminance, Luminance, Field of view, Tunnel

\section{Introduction}

Daylight can reduce the lighting demand of buildings[1]; however, it increases the need for tunnel lighting. When a driver passes into a tunnel rapidly during the daytime, the human eyes cannot adapt to the sharp change in luminance between inside and outside the tunnel, and temporary blindness will occur. The difference between access zone luminance $\left(L_{20}\right)$ and threshold zone luminance should be reduced to prevent this situation. Therefore, the International Commission on Illumination (CIE) proposed that the threshold zone in a tunnel should be equipped with enhanced lighting whose luminance is a certain ratio of access zone luminance. The transition zone lighting level should decrease gradually to make the human eyes gradually adapt from the high-illuminated threshold zone to the darker interior zone, and transition zone luminance is determined by $L_{20}$. Given that the luminance of the threshold and transition zones is much greater than that of the interior zone, the entire tunnel lighting energy consumption is concentrated in the threshold and transition zones (except for an ultralong tunnel). Thus, $L_{20}$ is an important parameter that determines the daily operating costs of tunnel lighting and the investment in lighting equipment.

CIE defines $L_{20}$ as the average luminance of a tunnel entrance seen by a driver at a specific point, which is located at the center of lanes, at the height of $1.5 \mathrm{~m}$, and at a distance from the tunnel portal equal to the stopping distance. The average luminance is taken from a 20-degree conical field of view, which is centered on one-quarter the height of the tunnel opening.

The traditional tunnel lighting design determines the installed power of lamps in accordance with the maximum $L_{20}$ (cumulative frequency) in a year. With the maximum $L_{20}$ as the basis for tunnel lighting operation, tunnels are often overlit. With the maturity of adjustable LED and intelligent control technology, the luminance of the threshold and transition zones can be adjusted in real time in accordance with $L_{20}$, which realizes energy saving in the tunnel while ensuring driving safety. The premise of tunnel intelligent dimming is that $L_{20}$ can be rapidly predicted. However, many influencing factors exist for $L_{20}$, which causes great difficulties in the prediction of $L_{20}$.

On this basis, scholars have conducted studies on the influence of landscape color, portal orientation, the percentage of objects in the 20-degree field of view, and test time on $L_{20}$ [2-6]. Nevertheless, studies in the past were still at the stage of qualitative analysis, and no $L_{20}$ prediction model had been established. Therefore, accurately predicting $L_{20}$ and clarifying the functional relationship of $L_{20}$ with weather, test time, the location of the tunnel, the inclination angle of the tunnel portal, the tunnel portal orientations, the color of objects, and the percentage of objects are pressing issues.

To this end, this study used theoretically calculated global illuminance received by an object to predict the object surface luminance and then obtained the predicted $L_{20}$, providing a reference for intelligent dimming of tunnel lighting. 


\section{State of the art}

CIE first proposed the use of a luminance meter with a 20degree viewing angle to measure $L_{20}$ in 1984. In 1990, it clarified the definition and the determination method for $L_{20}$. In 2004, the perceived contrast method was proposed, while $L_{20}$ method was kept in annexes. Many scholars have conducted related studies on $L_{20}$ and the luminance of landscape around the entrance portal. Gueorgiev[7] proposed that a high $L_{20}$ value causes the energy consumption of tunnel lighting during the day in summer to be 9 times that of at night. Peña-García et al.[8] proposed that coefficient $\mathrm{k}$, the length of threshold and transition zones, and $L_{20}$ are minimized by restricting the maximum speed of road tunnels, resulting in a reduction in tunnel energy consumption and the number of lamps, but they did not mention the disadvantages of the above method. Onaygil et al.[2] presented measures to reduce $L_{20}$, such as rough and dark surfaces of tunnel portals, road paving, high retaining walls, and green plants, trees, and shrubs around the tunnel, to achieve the purpose of an energy-saving tunnel, but many conclusions of the study were based on computer simulations rather than measured data. García-Trenas et al.[3] suggested measures to decrease $L_{20}$, including planting lowreflectivity ivy around the tunnel entrance in different climates, such as the Alps and the Mediterranean, and installing colored panels with simulated vegetation patterns to block the sky in the 20-degree field of view. In spite of this, ivy was not tested in other climates, and the species characteristics that were most suitable for tunnels in other climates were not determined. Peña-García et al.[9] proposed to install black solar panels around a tunnel, which not only provides power for the tunnel facilities (lighting, ventilation, emergency) but also reduces $L_{20}$, which significantly saves energy consumption, carbon dioxide emissions, and investment in lighting installations. However, no profit algorithm for installing solar panels based on the direction of the tunnel and the surrounding terrain was developed. López et al.[10] presented that when the lamps of an open traffic tunnel need to be updated or maintained, vehicle-based images can be used to obtain $L_{20}$, without the need to take photos from the stopping distance to improve test safety, but the study ignored that objects in the 20degree field of view of a driver may be different for various distances. Blaser et al.[11] proposed that the safe maximum $L_{20}$ be obtained from the cumulative frequency distribution of $L_{20}$ throughout a year on the basis of the known empirical relationship between luminance and daylight illuminance and the average relative sunshine time distribution in the relevant area during a year, but the proposed method had poor accuracy. Pachamanov et al.[12] obtained the safe maximum $L_{20}$ with different orientations of the entrance portal in Bulgaria. Doulos et al.[13] calculated $L_{20}$ in 11 existing tunnels and used the traffic weighting method to calculate threshold zone luminance, thereby reducing the luminance curve of the transition zone. Nonetheless, the proposed method was not combined with traffic detection sensors because the traffic volume can determine tunnel class and thus the lighting needs. Bouroussis et al.[4] investigated that the lateral position of $L_{20}$ instrument would cause a 20-degree field of view difference between a driver and the instrument and studied the effect of the field of view difference on $L_{20}$ for different orientations. However, the study only considered four orientations, i.e., east, west, south, and north; more orientations were not studied. Xu et al.[14] introduced the research idea of the dynamic characteristics of access zone luminance and believed that the dynamic change of access zone luminance is essentially formed by the change in the illuminance received by objects outside a tunnel, but the law of dynamic change of access zone luminance was not studied. Zhao et al.[5, 6] analyzed the changing law of $L_{20}$ with time under different tunnel portal orientations. Nevertheless, they did not study the changing law of $L_{20}$ with tunnel portal orientations and weather. Deng et al.[15] established the relationship between the luminance of landscape outside a tunnel and horizontal illumination through measurements and proposed a new method to determine the luminance of landscape outside the tunnel. Zhang[16] used the radiation data of a weather station and an established irradiance-illuminance conversion model to obtain vertical illuminance, then the luminance of scenery around a tunnel was determined in combination with the reflection coefficient. He et al.[17] used an SLR digital camera to test $L_{20}$ and improved a post-processing method for $L_{20}$. The proposed post-processing method increased the efficiency by more than 10 times, and the accuracy of $L_{20}$ was also increased. Nevertheless, the digital camera was only calibrated outdoors with a luminance meter, without using a better integrating sphere. Xiao[18] used an OV5640 camera calibrated with an imaging photometer to measure $L_{20}$, and a set of Linux-based luminance measurement systems was developed. Liang et al.[19] proposed that global horizontal irradiance and illuminance under a cloudless sky are linear functions of solar altitude angle, and a slope illuminance prediction method that does not rely on measured data was developed, laying the foundation for $L_{20}$ prediction under a cloudless sky. However, the proposed method only verified the predicted illuminance in mild climate zones, and the performance of the proposed method in polar, cold, arid, and equatorial climate zones was not studied.

The above analyses conducted studies on minimizing $L_{20}$, measuring $L_{20}$, and obtaining the safe maximum $L_{20}$. Nonetheless, few studies exist on the prediction of $L_{20}$. The ratio of luminance to illuminance of a specific color object within a 20-degree field of view is an approximate constant. The global illuminance received by an object is predicted first, then the object surface luminance is calculated. $L_{20}$ is predicted last in accordance with the percentage of objects in the 20-degree field of view. The predicted object surface luminance and $L_{20}$ are verified with the measured values, and the reasons for errors for predicting $L_{20}$ are analyzed.

The rest of this study is organized as follows. The third section establishes $L_{20}$ forecasting method system. The fourth section verifies the predicted values through measurements. The fifth section draws the conclusion of this study.

\section{Methodology}




\subsection{Prediction of solar position}

Daylight includes direct sunlight and sky light. Objects outside a tunnel reflect daylight to form object luminance. The average object luminance in the 20-degree field of view received by a driver at the stopping distance outside the tunnel is $L_{20}$. The solar position (the solar elevation, zenith, and azimuth) should be obtained to predict $L_{20}$. The solar altitude angle is obtained using the following formula (1)[20]:

$A_{\mathrm{as}}=\frac{180}{\pi} \arcsin \left(\sin l_{a} \sin D_{s}+\cos l_{a} \cos D_{s} \cos A_{i s}\right)$

where $A_{a s}$ is the angle of elevation of the sun above the horizon (degrees); $\pi$ is the pi; $l_{a}$ is the geographic latitude (degrees), $D_{s}$ is the sun declination angle (degrees), which is calculated using Formula (2); and $A_{t s}$ is the solar hour angle (degrees), which is expressed in Formula (5).

$D_{s}=0.3723-0.758 \cos \widetilde{\sigma}+0.3656 \cos (2 \mho)+0.0201 \cos (3 \mho)$

$+23.2567 \sin \mho+0.1149 \sin (2 \mho)-0.1712 \sin (3 \mho)$

where $\mho$ (radians) is calculated as

$\mho=0.0172\left(N_{x}-N_{2}\right)$

where $N_{x}$ is the day of a year (dimensionless). For example, February 1 is the 32 nd day of the year, that is, $N_{x}=32$.

$N_{2}=0.2422 y-401.0906-\operatorname{INT}\left(\frac{y-1985}{4}\right)$

where $y$ represents the year, and INT is a function that returns an integer.

The solar hour angle is expressed as follows:

$A_{i s}=15 t_{s}-180$

where $t_{s}$ is the solar time (hours), which is obtained as

$t_{s}=\left\{\begin{array}{lll}t_{l o c}+\frac{15\left|z_{t}\right|-L_{l o c}}{15}+\vartheta & \text { Western } & \text { Hemisphere } \\ t_{l o c}-\frac{15\left|z_{t}\right|-L_{l o c}}{15}+\vartheta & \text { Eastern } & \text { Hemisphere }\end{array}\right.$

where $t_{l o c}$ is the local standard time (hours), $z_{t}$ is the time zone (dimensionless), and $L_{l o c}$ is the local longitude (degrees).

$\vartheta=\frac{0.0028-1.9857 \sin \mho+9.9059 \sin (2 \mho)-7.0924 \cos \mho-0.6882 \cos (2 \mho)}{60}$

The angular distance between the sun and zenith $A_{z s}$ (radians) is expressed as follows:

$A_{z s}=\frac{\pi}{2}-\frac{\pi}{180} A_{a s}$
The azimuth of the sun (clockwise from the north) is obtained using the following formula[20]:

$A Z_{s}= \begin{cases}\pi-\arccos \frac{\sin A_{a s} \sin l_{a}-\sin D_{s}}{\cos A_{a s} \cos l_{a}} & t_{s} \leq 12 \\ \pi+\arccos \frac{\sin A_{a s} \sin l_{a}-\sin D_{s}}{\cos A_{a s} \cos l_{a}} & t_{s}>12\end{cases}$

where $A Z_{s}$ is the azimuth of the sun (radians).

3.2 Prediction of global illuminance received by an object The global illuminance received by an object is an important physical quantity for predicting $L_{20}$, which is expressed as follows:

$I_{\beta g}=I_{\beta d}+I_{\beta s}+I_{\beta r}$

where $I_{\beta \mathrm{g}}$ is the global illuminance received by the object (lux), $I_{\beta d}$ is the diffuse illuminance received by the object (lux), $I_{\beta s}$ is the direct illuminance received by the object (lux), and $I_{\beta r}$ is the reflected illuminance received by the object (lux).

$I_{\beta d}$ is calculated using the Perez model[21, 22], which is the point source simplified version, as shown as follows:

$I_{\beta d}=I_{h d}\left[0.5(1+\cos \beta)\left(1-F_{s}\right)+F_{s}(a / b)+F_{h} \sin \beta\right]$

where $I_{h d}$ is the diffuse illuminance in the horizontal plane (lux); $\beta$ is the inclination angle of the object surface (degrees); $F_{s}$ and $F_{h}$ are the anisotropy coefficients (dimensionless) of circumsolar and the horizon, respectively, which are expressed in Formulas (18) and (19). The calculations of $a$ and $b$ are shown in Formulas (25) and (26).

$$
I_{h d}=I_{h g}-I_{i s} \sin A_{a s}
$$

where $I_{h g}$ is the global illuminance in the horizontal plane (lux), and $I_{\text {is }}$ is the normal incidence direct illuminance (lux). Under a cloudless sky, $I_{h g}$ is expressed as follows[19]:

$I_{h g}=1486.667 A_{a s}$

$I_{i s}$ is obtained using the following formula[23]:

$I_{i s}=I_{e x} \cdot f_{c} \cdot \exp \left(-m \cdot f_{e} \cdot f_{I}\right)$

where $I_{\mathrm{ex}}$ is the extraterrestrial illuminance, which is 133800 lux. $f_{c}$ is the date correction (dimensionless) of extraterrestrial illuminance constant, $m$ is the relative air mass (dimensionless), and $f_{e}$ is the luminous extinction (dimensionless) under dry and clean air, which are expressed in Formulas (15)[24], (16)[25], and (17)[25], respectively. $f_{I}$ is the luminous turbidity factor (dimensionless). For a cloudless sky, the value of $f_{I}$ is $2.5[26]$. 
$f_{c}=1+0.0337 \cos \left[0.9863\left(N_{x}-4\right)\right]$

$m=\frac{1}{\sin A_{a s}+0.50572\left(A_{a s}+6.07995\right)^{-1.6364}}$

$f_{e}=\frac{1}{10.1+0.045 m}$

$F_{s}$ and $F_{h}$ in Formula (11) are expressed as

$$
F_{s}=f_{s 1}+f_{s 2} \Delta+f_{s 3} A_{z s}
$$

$F_{h}=f_{h 1}+f_{h 2} \Delta+f_{h 3} A_{z s}$

where $f_{s 1}, f_{s 2}, f_{s 3}, f_{h 1}, f_{h 2}$, and $f_{h 3}$ are the illuminance coefficients (dimensionless) of the point source simplified Perez model, as shown in Table 1.

Table. 1. Illuminance coefficients of the point source simplified Perez model

\begin{tabular}{l|l|l|l|l|l|l|l}
\hline $\boldsymbol{\varepsilon}$ & $\begin{array}{l}\text { Upper } \\
\text { bound }\end{array}$ & $\boldsymbol{f}_{\boldsymbol{s} 1}$ & $\boldsymbol{f}_{\boldsymbol{s} \mathbf{2}}$ & $\boldsymbol{f}_{\boldsymbol{s} \mathbf{3}}$ & $\boldsymbol{f}_{\boldsymbol{h} \boldsymbol{1}}$ & $\boldsymbol{f}_{\boldsymbol{h} \mathbf{2}}$ & $\boldsymbol{f}_{\boldsymbol{h} \mathbf{3}}$ \\
\hline 1 & 1.065 & 0.011 & 0.57 & -0.081 & -0.095 & 0.158 & -0.018 \\
2 & 1.23 & 0.429 & 0.363 & -0.307 & 0.05 & 0.008 & -0.065 \\
3 & 1.5 & 0.809 & -0.054 & -0.442 & 0.181 & -0.17 & -0.092 \\
4 & 1.95 & 1.014 & -0.252 & -0.531 & 0.275 & -0.35 & -0.096 \\
5 & 2.8 & 1.282 & -0.42 & -0.689 & 0.38 & -0.56 & -0.114 \\
6 & 4.5 & 1.426 & -0.653 & -0.779 & 0.425 & -0.79 & -0.097 \\
7 & 6.2 & 1.485 & -1.214 & -0.784 & 0.411 & -0.63 & -0.082 \\
8 & - & 1.17 & -0.3 & -0.615 & 0.518 & -1.89 & -0.055 \\
\hline
\end{tabular}
as

The brightness of the sky (dimensionless), $\Delta$, is defined

$$
\Delta=\frac{m E_{h d}}{E_{\mathrm{e} x}}
$$

where $E_{h d}$ is the diffuse irradiance in the horizontal plane $\left(\mathrm{W} / \mathrm{m}^{2}\right)$; and $E_{\mathrm{e} x}$ is the extraterrestrial irradiance, which is $1366 \mathrm{~W} / \mathrm{m}^{2}$.

$E_{h d}=E_{h g}-E_{i s} \sin A_{a s}$

where $E_{h g}$ is the global irradiance in the horizontal plane $\left(\mathrm{W} / \mathrm{m}^{2}\right)$; under a cloudless sky, it can be obtained using Formula (22)[19]. $E_{i s}$ is the normal incidence direct irradiance $\left(\mathrm{W} / \mathrm{m}^{2}\right)$, and it can be obtained using Formula (23) under a cloudless sky[27].

$$
\begin{aligned}
& E_{h g}=15.178 A_{a s} \\
& E_{i s}=1353 * 0.7^{m^{0.678}}
\end{aligned}
$$

The clearness of the sky (dimensionless) in Table 1., $\varepsilon$, is defined as

$$
\varepsilon=\frac{1.041 A_{z s}^{3}+\frac{E_{i s}+E_{h d}}{E_{h d}}}{1+1.041 A_{z s}^{3}}
$$

$a$ and $b$ in Formula (11) are expressed as follows:

$a=\max \left(0, \cos A_{\beta i}\right)$

$b=\max \left(0.087, \cos A_{z s}\right)$

where $A_{\beta i}$ is the angle of incidence on the object surface, and it is obtained as follows:

$\cos A_{\beta i}=\sin A_{a s} \cos \beta+\cos A_{a s} \sin \beta \cos \left(\psi-A Z_{s}\right)$

where $\psi$ is the azimuth (radians) that the object surface faces.

The direct illuminance received by the object is determined by $I_{i s}$ and $A_{\beta i}$, as shown as follows:

$I_{\beta s}=I_{i s} \cos A_{\beta i}$

The reflected illuminance received by the object is expressed as

$I_{\beta r}=0.5 f_{g} I_{h g}(1-\cos \beta)$

where $f_{g}$ is the albedo coefficient (dimensionless). In the absence of measured data, the value of $f_{g}$ is $0.2[28]$.

3.3 Proposed prediction model for object surface luminance

Assuming that the daylight received by objects within a 20 degree field of view is uniformly reflected in all directions, the ratio of luminance to illuminance of a specific color object is constant, and this ratio is denoted as $r$. This law will be confirmed in Section 4. The luminance of the object can be expressed as

$L=I_{\beta g} \cdot r$

where $L$ is the object surface luminance.

\subsection{Proposed prediction model for $L_{20}$}

In consideration of the different colors of objects in the 20degree field of view, this field of view is divided into $n$ regions, then $L_{20}$ is expressed as follows:

$L_{20}=\sum_{i=1}^{i=n} L_{i} p_{i}$

where $L_{i}$ is the luminance of the $i$-th color object, and $p_{i}$ is the percentage of the $i$-th color object in the 20-degree field of view.

$p_{1}+p_{2}+\cdots+p_{n}=1$

The above 32 formulas should be programmed to improve the prediction efficiency of $L_{20}$. The input 
parameters of the prediction $L_{20}$ program include the test date, $t_{l o c}, z_{t}, L_{l o c}, l_{a}, \beta, \psi, r$, and $p_{i}$. The above input parameters are all factors that affect $L_{20}$. Therefore, all influencing factors have been quantified in the proposed $L_{20}$ prediction method.

\section{Result Analysis and Discussion}

\subsection{Ratio of normal luminance to illuminance}

Normal luminance and illuminance on object surface were tested. The tested objects included a horizontal asphalt pavement and vertical cards made of tender green and dark green leaves. The test site was located at 104.41 degrees east longitude and 31.09 degrees north latitude. The luminance meter (LS-150, Konica Minolta, Tokyo, Japan) and illuminance meter (TES-1339R, TES Electrical Electronic Corp., Taipei, Taiwan) were calibrated before use. The results in Tables 2-4 show that the ratio of normal luminance to illuminance on the surface of a specific color object is approximately constant. The test time and weather do not affect this ratio, but the object color is the decisive factor for this ratio.

Table 2. Ratio of normal luminance to illuminance on a horizontal asphalt pavement

\begin{tabular}{|c|c|c|c|c|c|}
\hline Date & Time & Weather & $\begin{array}{l}L_{R n} \\
\left(\mathrm{~cd} / \mathrm{m}^{2}\right)\end{array}$ & $\begin{array}{l}I_{R} \\
\text { (lux) }\end{array}$ & $\begin{array}{l}\frac{L_{R n}}{I_{R}} \\
\text { (cd/lm) }\end{array}$ \\
\hline \multirow{8}{*}{$5 / 27 / 2020$} & $9: 35$ & \multirow{8}{*}{ Overcast } & 310.2 & 4136 & 0.075 \\
\hline & $10: 22$ & & 398.7 & 5462 & 0.073 \\
\hline & $11: 23$ & & 527.1 & 7123 & 0.074 \\
\hline & $12: 45$ & & 661.0 & 9180 & 0.072 \\
\hline & $13: 10$ & & 685.4 & 9019 & 0.076 \\
\hline & $14: 20$ & & 794.2 & 11680 & 0.068 \\
\hline & $15: 15$ & & 500.8 & 7475 & 0.067 \\
\hline & $17: 13$ & & 814.9 & 11810 & 0.069 \\
\hline \multirow{9}{*}{$5 / 29 / 2020$} & $8: 49$ & \multirow{9}{*}{ Clear } & 3567 & 50240 & 0.071 \\
\hline & $9: 49$ & & 5128 & 69300 & 0.074 \\
\hline & $10: 50$ & & 6380 & 88610 & 0.072 \\
\hline & $11: 50$ & & 7682 & 106700 & 0.072 \\
\hline & $12: 59$ & & 8259 & 119700 & 0.069 \\
\hline & 13:31 & & 8606 & 116300 & 0.074 \\
\hline & $14: 29$ & & 7366 & 100900 & 0.073 \\
\hline & $15: 35$ & & 5713 & 80470 & 0.071 \\
\hline & $16: 44$ & & 4273 & 58540 & 0.073 \\
\hline
\end{tabular}

$L_{R n}$ and $I_{R}$ are the normal luminance and illuminance on a horizontal asphalt pavement, respectively.

Table 3. Ratio of normal luminance to illuminance on a vertical card made of a tender green leaf

\begin{tabular}{|c|c|c|c|c|c|}
\hline Date & Time & Weather & $\begin{array}{l}L_{T G v n} \\
\left(\mathrm{~cd} / \mathrm{m}^{2}\right)\end{array}$ & $\begin{array}{c}I_{T G v} \\
\text { (lux) }\end{array}$ & $\begin{array}{l}\frac{L_{T G v n}}{I_{T G v}} \\
\text { (cd/lm) }\end{array}$ \\
\hline \multirow{7}{*}{$12 / 24 / 2020$} & $14: 45$ & \multirow{7}{*}{ Overcast } & 511.2 & 8960 & 0.057 \\
\hline & $15: 27$ & & 293.8 & 5890 & 0.050 \\
\hline & $15: 45$ & & 256.1 & 4648 & 0.055 \\
\hline & $16: 17$ & & 206.2 & 3656 & 0.056 \\
\hline & $16: 39$ & & 136.7 & 2673 & 0.051 \\
\hline & $17: 07$ & & 106.3 & 2139 & 0.050 \\
\hline & $17: 27$ & & 96.13 & 1757 & 0.055 \\
\hline \multirow{6}{*}{$12 / 23 / 2020$} & $13: 36$ & \multirow{6}{*}{ Clear } & 979.4 & 18480 & 0.053 \\
\hline & $14: 00$ & & 1368 & 26820 & 0.051 \\
\hline & $14: 25$ & & 1960 & 35000 & 0.056 \\
\hline & $14: 52$ & & 2277 & 42960 & 0.053 \\
\hline & $15: 16$ & & 2544 & 48930 & 0.052 \\
\hline & $15: 46$ & & 2867 & 54100 & 0.053 \\
\hline
\end{tabular}

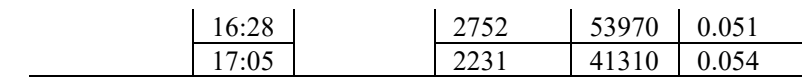

$L_{T G v n}$ and $I_{T G v}$ are the normal luminance and illuminance on a vertical card made of a tender green leaf, respectively.

Table 4. Ratio of normal luminance to illuminance on a vertical card made of a dark green leaf

\begin{tabular}{|c|c|c|c|c|c|}
\hline Date & Time & Weather & $\begin{array}{c}L_{D G v n} \\
\left(\mathrm{~cd} / \mathrm{m}^{2}\right)\end{array}$ & $\begin{array}{c}I_{D G v} \\
\text { (lux) }\end{array}$ & $\begin{array}{l}\frac{L_{D G v n}}{I_{D G v}} \\
\text { (cd/lm) }\end{array}$ \\
\hline \multirow{7}{*}{$12 / 24 / 2020$} & $14: 35$ & \multirow{7}{*}{ Overcast } & 100.6 & 5826 & 0.017 \\
\hline & $15: 05$ & & 84.13 & 5162 & 0.016 \\
\hline & $15: 37$ & & 58.88 & 3324 & 0.018 \\
\hline & $16: 00$ & & 52.97 & 3228 & 0.016 \\
\hline & $16: 27$ & & 53.48 & 2933 & 0.018 \\
\hline & $17: 00$ & & 41.19 & 2247 & 0.018 \\
\hline & $17: 17$ & & 39.06 & 2197 & 0.018 \\
\hline \multirow{12}{*}{$12 / 23 / 2020$} & $13: 46$ & \multirow{12}{*}{ Clear } & 1323 & 77850 & 0.017 \\
\hline & $13: 58$ & & 1310 & 77040 & 0.017 \\
\hline & $14: 11$ & & 1215 & 75950 & 0.016 \\
\hline & $14: 34$ & & 1324 & 73570 & 0.018 \\
\hline & $15: 02$ & & 1184 & 69650 & 0.017 \\
\hline & $15: 18$ & & 1137 & 66860 & 0.017 \\
\hline & $15: 28$ & & 1103 & 64890 & 0.017 \\
\hline & $15: 42$ & & 1113 & 61820 & 0.018 \\
\hline & $15: 56$ & & 990.9 & 58290 & 0.017 \\
\hline & $16: 11$ & & 862.1 & 53880 & 0.016 \\
\hline & $16: 39$ & & 735.6 & 43270 & 0.017 \\
\hline & $17: 15$ & & 417.5 & 24560 & 0.017 \\
\hline
\end{tabular}

$L_{D G v n}$ and $I_{D G v}$ are the normal luminance and illuminance on a vertical card made of a dark green leaf, respectively.

\subsection{Effect of viewing angle on object surface luminance}

In the 20-degree field of view, a driver's eyes receive object surface luminance, which is at different viewing distances and angles. Given that the viewing distance does not affect object surface luminance, the object surface luminance at different viewing angles was studied. Both LS-150 luminance meters measured an object's luminance at the same time, one of which measured the normal luminance (0degree viewing angle), and the other one measured the luminance at a 72-degree viewing angle. The luminance on a horizontal asphalt pavement, a vertical card made of a tender green leaf, and a vertical card made of a dark green leaf at a 72-degree viewing angle are denoted as $L_{R 72}, L_{T G v 72}$, and $L_{D G v 72}$, respectively. Figures 1-3 demonstrate that the luminance at the 72-degree viewing angle is close to its normal luminance. Figures 4-6 show that the relative error between the luminance at the 72-degree viewing angle and its normal luminance is $-10 \%--4 \%$ and $4 \%-10 \%$. This relative error exceeds $\pm 2 \%$, which is the uncertainty of the LS-150 luminance meter, such that the luminance difference at different viewing angles is not caused by the measurement error of the LS-150 luminance meter. Therefore, the tested objects reflect daylight approximately evenly in all directions. To simplify the prediction of $L_{20}$, it is necessary to assume that the luminance of an object within the 20degree field of view is the same at all viewing angles. Given that the ratio of normal luminance to the illuminance on the surface of a specific color object is approximately a fixed value, it can be assumed that the ratio of luminance to the illuminance on the surface of a specific color object at any viewing angle is constant when predicting $L_{20}$. 


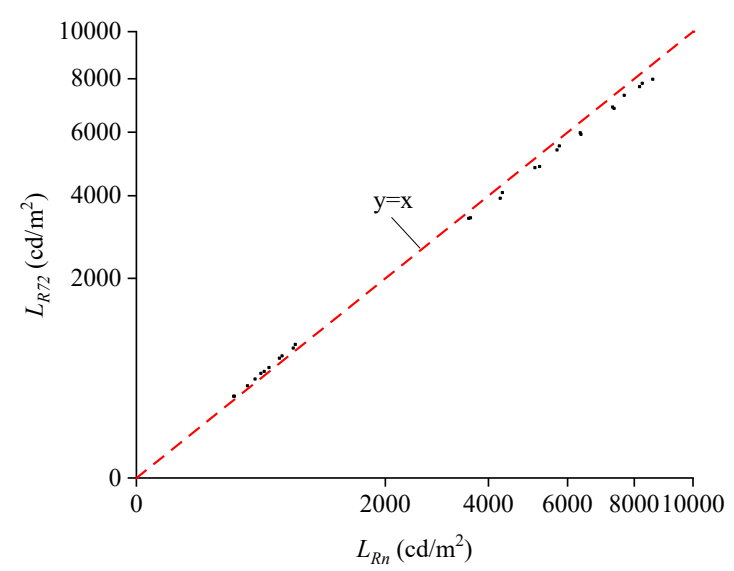

Fig. 1. Scatterplot of $L_{R n}$ vs. $L_{R 72}$

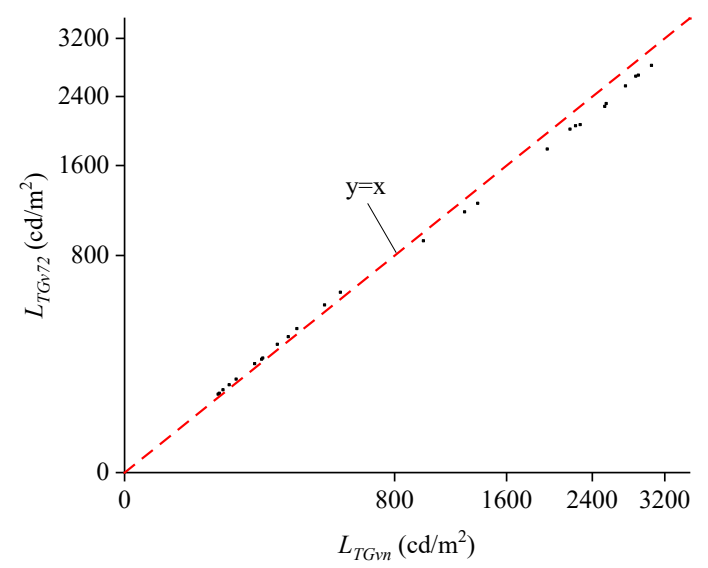

Fig. 2. Scatterplot of $L_{T G v n}$ vs. $L_{T G v 72}$

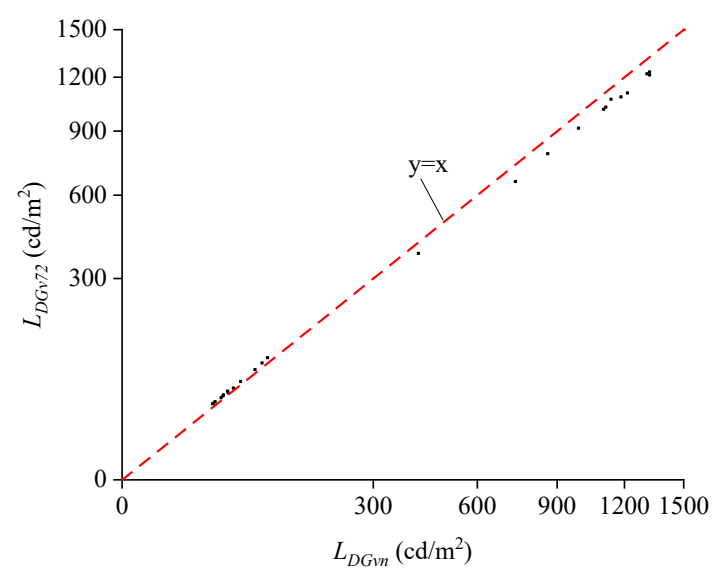

Fig. 3. Scatterplot of $L_{D G v n}$ vs. $L_{D G v 72}$

\subsection{Object surface luminance verification}

Object surface luminance is the basis for predicting $L_{20}$. In this study, the luminance of the horizontal asphalt pavement and the vertical cards facing east, west, south, and north was measured every $10 \mathrm{~min}$. The cards were made of tender green leaves. The above five luminance are denoted as $L_{R}, L_{T G e v}, L_{T G w v}, L_{T G s v}$, and $L_{T G n v}$, respectively. The test site was located at 104.41694 degrees east longitude and 31.09056 degrees north latitude. The test time was 10:4514:55 on July 2, 2020. The sky was cloudless during the test. Figures 7-11 show the comparison results of the measured and predicted values of object surface luminance. The predicted luminance is close to the measured values, except for $L_{T G n v}$.

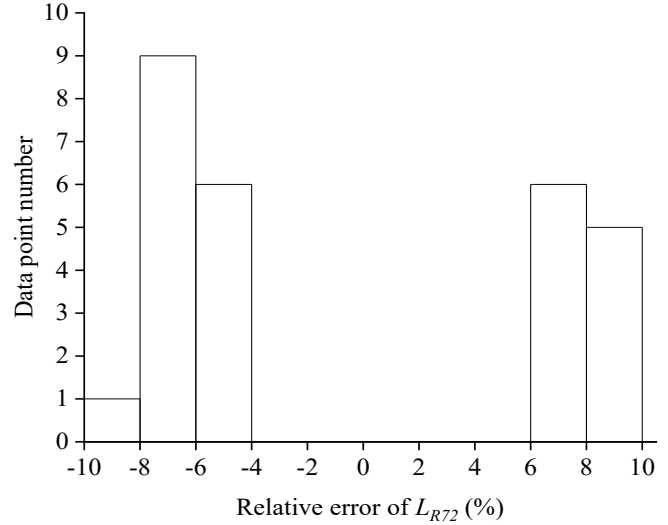

Fig. 4. Relative error histogram of $L_{R 72}$

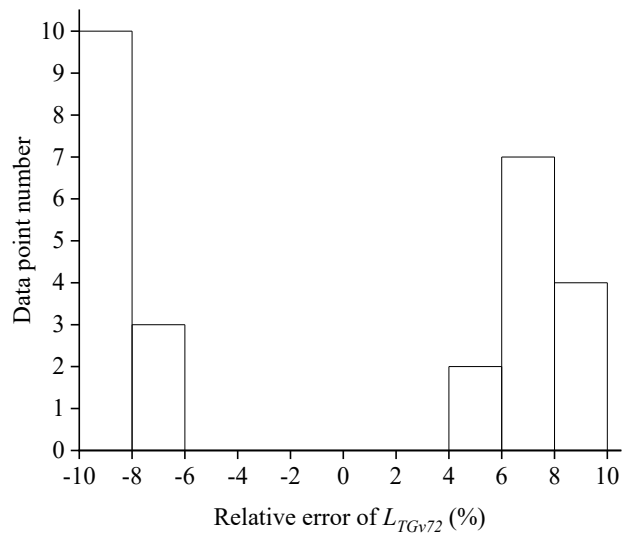

Fig. 5. Relative error histogram of $L_{T G v 72}$

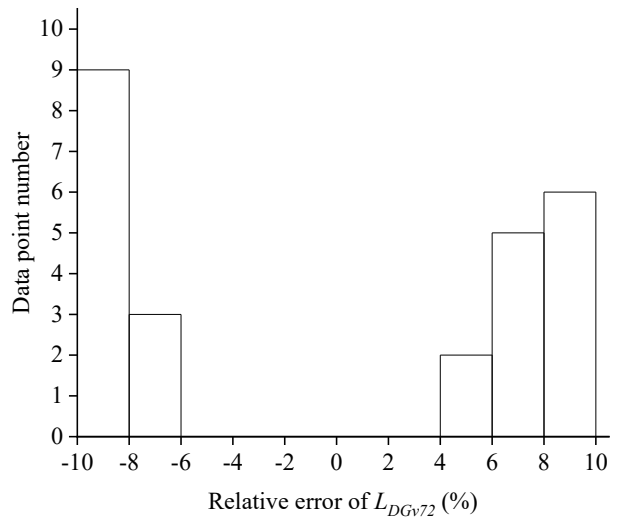

Fig. 6. Relative error histogram of $L_{D G v 72}$

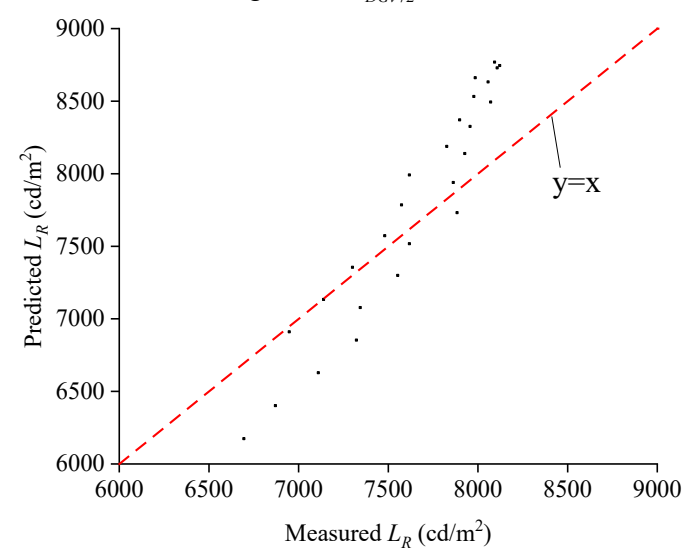

Fig. 7. Scatterplot of the measured $L_{R}$ vs. the predicted $L_{R}$ 


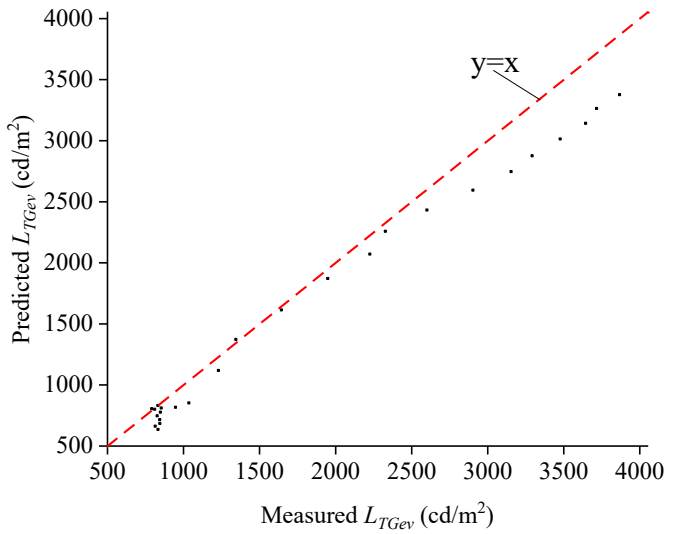

Fig. 8. Scatterplot of the measured $L_{T G e v}$ vs. the predicted $L_{T G e v}$

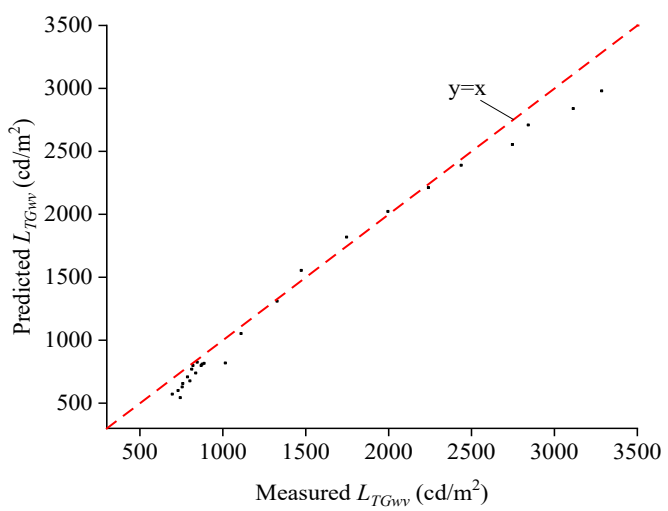

Fig. 9. Scatterplot of the measured $L_{T G w v}$ vs. the predicted $L_{T G w v}$

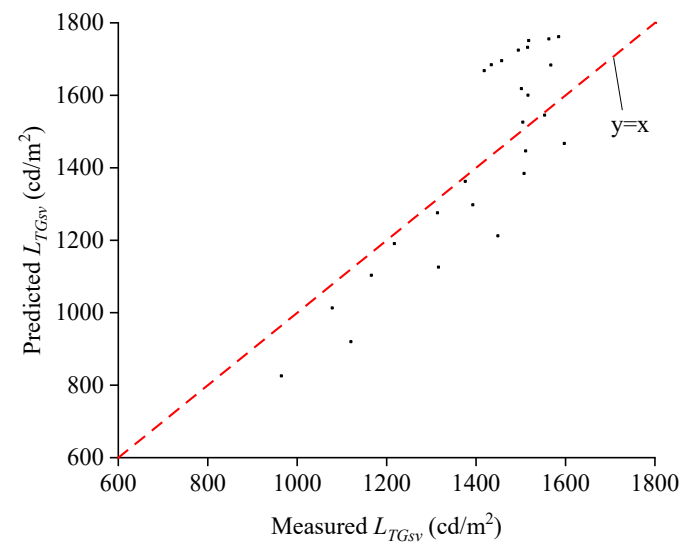

Fig. 10. Scatterplot of the measured $L_{T G s v}$ vs. the predicted $L_{T G s v}$

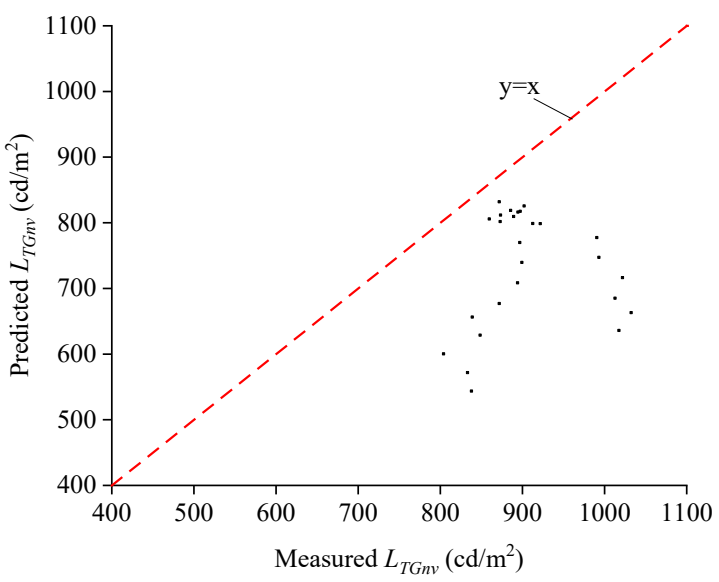

Fig. 11. Scatterplot of the measured $L_{T G n v}$ vs. the predicted $L_{T G n v}$
Figures 12-16 show the relative error frequency distribution of $L_{R}, L_{T G e v}, L_{T G w v}, L_{T G s v}$, and $L_{T G n v}$. The relative errors of $L_{R}, L_{T G e v}, L_{T G W v}, L_{T G s v}$, and $L_{T G w v}$ are $-10 \%-10 \%$, $-30 \%-10 \%, \quad-30 \%-10 \%, \quad-20 \%-20 \%, \quad$ and $-40 \%-0$, respectively. The relative error of $L_{R}$ is the smallest, whereas the relative error of $L_{T G n v}$ is the largest.

Relative error $=\frac{(P-M)}{M} \times 100 \%$

where $P$ denotes the predicted data, and $M$ denotes the measured data.

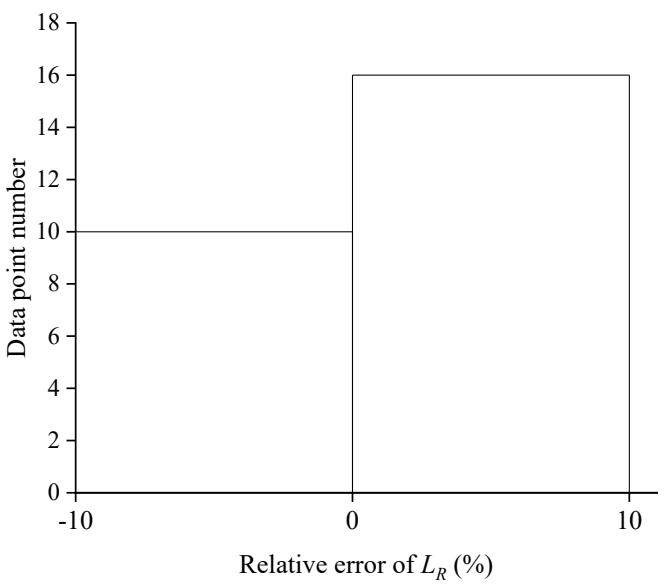

Fig. 12. Relative error histogram of $L_{R}$

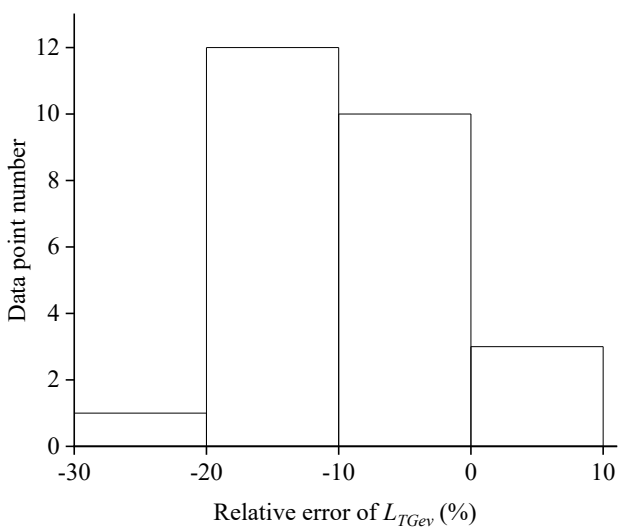

Fig. 13. Relative error histogram of $L_{T G e v}$

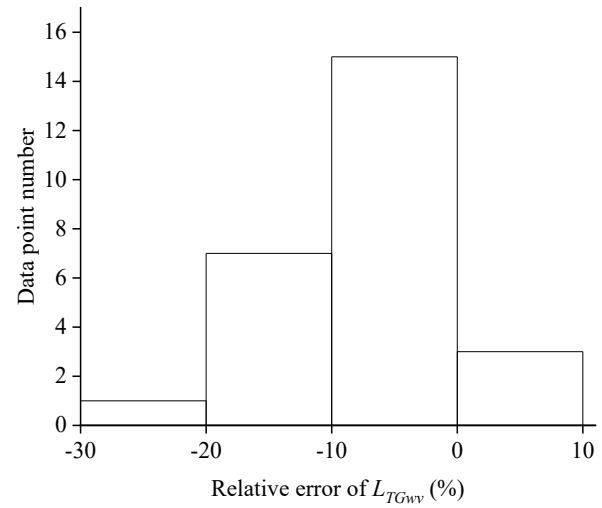

Fig. 14. Relative error histogram of $L_{T G w v}$ 


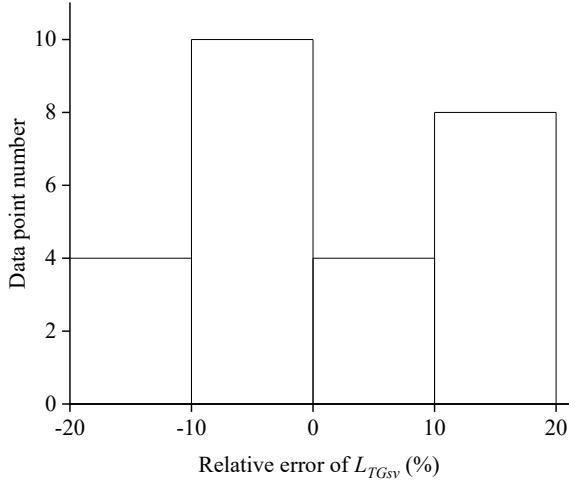

Fig. 15. Relative error histogram of $L_{T G s v}$

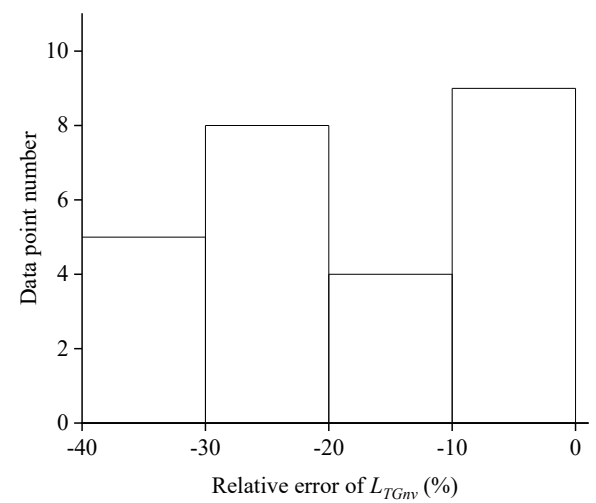

Fig. 16. Relative error histogram of $L_{T G n v}$

The relative root-mean-square error (RRMSE) and relative mean bias error (RMBE) in statistics were used to evaluate the overall error of the forecast data. Table 5 shows the overall error of $L_{R}, L_{T G e v}, L_{T G w v}, L_{T G s v}$, and $L_{T G n v}$. The RMBEs of $L_{T G e v}, L_{T G w v}$, and $L_{T G n v}$ are less than 0 , indicating that the predicted values of $L_{T G e v}, L_{T G w v}$, and $L_{T G n v}$ are generally small. From Table 5, the overall error of $L_{R}$ is the smallest, and that of $L_{T G n v}$ is the largest. The measured $L_{R}, L_{T G e v}, L_{T G w v}, L_{T G s v}$, and $L_{T G n v}$ and the corresponding predicted values are generally in good agreement, indicating that the assumptions in Formulas 10 30 are appropriate.

$$
R R M S E=\frac{\sqrt{\frac{\sum_{1}^{n}(P-M)^{2}}{n}}}{M} \times 100 \%
$$

where $n$ is the amount of data, and $\bar{M}$ is the average measured value.

$$
R M B E=\frac{\sum_{1}^{n}(P-M)}{n \cdot \bar{M}} \times 100 \%
$$

Table 5. Overall error of object surface luminance

\begin{tabular}{l|l|l|l|l|l}
\hline & $L_{R}$ & $L_{T G e v}$ & $L_{T G w v}$ & $L_{T G s v}$ & $L_{T G n v}$ \\
\hline RRMSE (\%) & 5.4 & 13.5 & 9.1 & 11.2 & 22.2 \\
\hline RMBE (\%) & 1.8 & -10.0 & -6.4 & 2.0 & -19.2 \\
\hline
\end{tabular}

\section{4 $L_{20}$ verification}

On May 29, 2020, $L_{20}$ of the tunnel located at 104.41785 degrees east longitude and 31.090786 degrees north latitude (the time zone is the eighth zone of the eastern hemisphere) was measured. The sky was cloudless during the test. $L_{20}$ test equipment was an imaging photometer (IC-PMY16, Radiant Vision Systems, Redmond, WA, USA). The tested tunnel portal was perpendicular to the asphalt pavement, and the azimuth that the portal faces was 257 degrees. The 20 degree field of view when the driver approached the tunnel is shown in Figure 17. The 20-degree field of view was divided into four different color areas, namely, light gray wall, black threshold zone, gray asphalt road, and tender green leaves. In accordance with the percentage of the four areas in the 20-degree field of view, $L_{20}$ can be expressed as

$L_{20}=0.303 L_{R}+0.016 L_{T G}+0.013 L_{W}+0.668 L_{t h}$

where $L_{T G}$ is the luminance of the tender green leaves, $L_{W}$ is the luminance of the light gray wall, and $L_{t h}$ is the luminance of the threshold zone.

Given that $L_{t h}$ is much smaller than the luminance of other areas and $L_{t h}$ can be ignored, Formula (36) can be simplified as

$L_{20}=0.303 L_{R}+0.016 L_{T G}+0.013 L_{W}$

The measured $r$ values of the gray asphalt pavement, tender green leaves, and light gray wall are $0.072,0.053$, and 0.121 , respectively.

The predicted value of $L_{20}$ was compared with the measured value in Table 6 and Figure 18. Figure 19 presents that the relative error of $L_{20}$ is $0-30 \%$. The RRMSE and RMBE of $L_{20}$ are $11.6 \%$ and $11.4 \%$, respectively. The predicted $L_{20}$ is slightly larger.

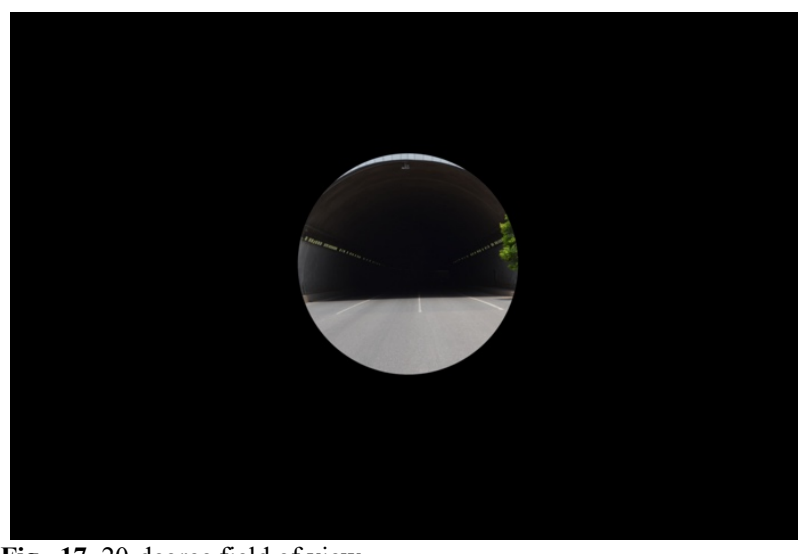

Fig. 17. 20-degree field of view

Table 6. Predicted and measured values of $L_{20}$

\begin{tabular}{c|c|c|c|c|c}
\hline Time & $\begin{array}{c}\text { Predicted } \\
L_{R} \\
\left(\mathrm{~cd} / \mathrm{m}^{2}\right)\end{array}$ & $\begin{array}{c}\text { Predicted } \\
L_{T G} \\
\left(\mathrm{~cd} / \mathrm{m}^{2}\right)\end{array}$ & $\begin{array}{c}\text { Predicted } \\
L_{W} \\
\left(\mathrm{~cd} / \mathrm{m}^{2}\right)\end{array}$ & $\begin{array}{c}\text { Predicted } \\
L_{20} \\
\left(\mathrm{~cd} / \mathrm{m}^{2}\right)\end{array}$ & $\begin{array}{c}\text { Measured } \\
L_{20} \\
\left(\mathrm{~cd} / \mathrm{m}^{2}\right)\end{array}$ \\
\hline $13: 00$ & 8623 & 1058 & 2416 & 2661 & 2467 \\
$13: 10$ & 8593 & 1314 & 3000 & 2664 & 2452 \\
$13: 20$ & 8511 & 1558 & 3557 & 2650 & 2413 \\
$13: 30$ & 8388 & 1821 & 4158 & 2625 & 2351 \\
$13: 40$ & 8235 & 2026 & 4625 & 2588 & 2387 \\
$13: 50$ & 8060 & 2215 & 5056 & 2543 & 2303 \\
$14: 00$ & 7870 & 2389 & 5454 & 2494 & 2234 \\
$14: 10$ & 7670 & 2550 & 5821 & 2440 & 2245
\end{tabular}




\begin{tabular}{l|l|l|l|l|l}
$14: 20$ & 7462 & 2699 & 6161 & 2384 & 2167 \\
$14: 30$ & 7248 & 2819 & 6436 & 2325 & 2102 \\
$14: 40$ & 7030 & 2953 & 6741 & 2265 & 1969 \\
$14: 50$ & 6809 & 3075 & 7021 & 2204 & 1975 \\
$15: 00$ & 6586 & 3188 & 7279 & 2141 & 1994 \\
$15: 10$ & 6361 & 3293 & 7518 & 2078 & 1829 \\
$15: 20$ & 6135 & 3390 & 7740 & 2014 & 1756 \\
$15: 30$ & 5908 & 3480 & 7945 & 1949 & 1741 \\
$15: 40$ & 5680 & 3564 & 8136 & 1884 & 1759 \\
$15: 50$ & 5451 & 3641 & 8312 & 1818 & 1588 \\
$16: 00$ & 5222 & 3712 & 8474 & 1752 & 1629 \\
$16: 10$ & 4993 & 3776 & 8622 & 1685 & 1487 \\
$16: 20$ & 4764 & 3834 & 8753 & 1619 & 1422 \\
$16: 30$ & 4535 & 3884 & 8868 & 1551 & 1437 \\
$16: 40$ & 4306 & 3926 & 8964 & 1484 & 1269 \\
$16: 50$ & 4077 & 3959 & 9038 & 1416 & 1205 \\
$17: 00$ & 3849 & 3980 & 9087 & 1348 & 1216 \\
$17: 10$ & 3621 & 3989 & 9108 & 1279 & 1060 \\
$17: 20$ & 3394 & 3984 & 9096 & 1210 & 989.6 \\
\hline
\end{tabular}

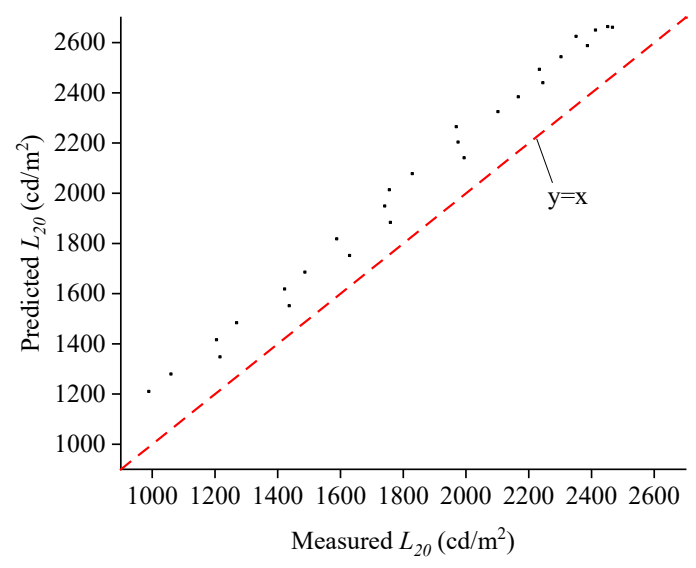

Fig. 18. Scatterplot of the measured $L_{20}$ vs. the predicted $L_{20}$

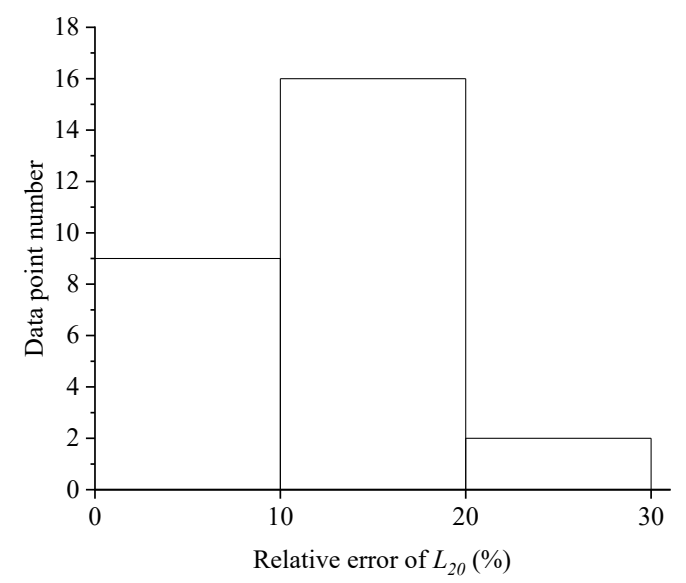

Fig. 19. Relative error histogram of $L_{20}$

\subsection{Discussion}

The proposed method can predict $L_{20}$ of any time under a cloudless sky, whereas the CIE method can only obtain the maximum $L_{20}$ in a year. The proposed method can predict $L_{20}$ with portals with different inclination angles, whereas the CIE method does not consider the impact of the inclination of the portal on $L_{20}$. The CIE method is limited to obtaining $L_{20}$ for four driving directions: east, west, south, and north. By contrast, the proposed method can predict $L_{20}$ for any driving direction.
The largest potential error in predicting $L_{20}$ comes from the prediction of $I_{\beta g}$. The used Perez point source model has two assumptions. One is that all the energy in the horizon zone is contained in an infinitely thin area with an elevation angle of 0 degree. The other is that all the circumsolar energy comes from one point. The above assumptions cause an error in predicting $I_{\beta d}$, especially when the surface cannot receive sunlight. but the Perez point source model is currently the most accurate model for predicting $I_{\beta d}$. The extraterrestrial irradiance and illuminance of the cloudless sky were assumed to reach the ground when the sun's altitude angle was 90 degrees in the used $I_{h g}, E_{h g}$ model proposed by Liang et al.[19]. The above assumption causes small errors in the prediction of $I_{h g}, E_{h g}$. In addition, the values of $f_{I}$ and $f_{g}$ affect the accuracy of $I_{i s}$ and $I_{\beta r}$, respectively. All the above errors cause bias in $I_{\beta \mathrm{g}}$.

Another source of error in predicting $L_{20}$ is $L$ obtained through $I_{\beta g}$. L within the 20-degree field of view is assumed to be the same at all viewing angles. Nevertheless, the objects in the 20-degree field of view reflect daylight approximately evenly in all directions. $L$ is slightly distinct at different viewing angles.

The 20-degree field of view was divided into multiple areas of different colors to calculate $L_{20}$. The premise of the proposed $L_{20}$ model is that the colors in the divided area are exactly the same. However, it can only be guaranteed that the colors in the segmented region are roughly the same when the 20-degree field of view is segmented, which causes errors in the predicted $L_{20}$.

\section{Conclusions}

This study proposed a new prediction model for object surface luminance to predict $L_{20}$ of road tunnels under a cloudless sky. A combination of theoretical research and measured data was used. The predicted object surface luminance and $L_{20}$ under a cloud-free sky were verified, and the reasons for the error were analyzed. In the end, the following conclusions were reached:

(1) Objects in the 20-degree field of view have approximately the same luminance at different viewing angles. It can be supposed that the ratio of luminance to illuminance on the surface of a specific color object is constant when predicting $L_{20}$. This ratio is not affected by the test time and weather, but the color is the decisive factor for the ratio.

(2) The proposed prediction method quantitatively considers all factors that affect $L_{20}$, such as weather, test time, the location of the tunnel, the inclination angle of the portal, the orientation of the portal, the color of objects around the portal, and the percentage of objects in the 20degree field of view. The function cannot be achieved in the CIE method.

(3) In the example, the RRMSE and RMBE of $L_{20}$ predicted using the proposed method are $11.6 \%$ and $11.4 \%$, respectively, which can meet the engineering requirements.

$L_{20}$ prediction method proposed in this study has good operability. The proposed method has reference significance 
for intelligent dimming research of highway tunnels. CIE recommends 15 sky types. $I_{\beta g}$ is extremely difficult to predict under cloud sky types. The color, distribution, and thickness of clouds should be considered. This study only carried out $L_{20}$ forecast of a cloudless sky. Predicting $L_{20}$ under a clouded sky will be the focus of future research.

\section{Acknowledgements}

This work was supported by the National Natural Science Foundation of China. (Approval number: 51878107)

This is an Open Access article distributed under the terms of the Creative Commons Attribution License.

\section{References}

1. Mavridou, T.,Doulos, L. T., "Evaluation of different roof types concerning daylight in industrial buildings during the initial design phase: Methodology and case study". Buildings, 9(7), 2019, pp.170.

2. Onaygil, S., Güler, Ö.,Erkin, E., "Determination of the effects of structural properties on tunnel lighting with examples from Turkey". Tunnelling and underground space technology, 18(1), 2003, pp.85-91.

3. García-Trenas, T., López, J.,Peña-García, A., "Proposal to forest Alpine tunnels surroundings to enhance energy savings from the lighting installations. Towards a standard procedure". Tunnelling and underground space technology, 78(1), 2018, pp.1-7.

4. Bouroussis, C. A., Nikolaou, D. T.,Topalis, F. V., "Optimization of tunnel lighting control by re-aiming of the external L20 luminance meter". In: CIE x046: 2019 Proceedings of 29th CIE Session, Washington DC, USA: CIE, 2019, pp.1595-1604.

5. Zhao, W., Liu, Q., Liu, S.,Wang, Q., "Study on Time-varying Characteristics of Outside Brightness of Highway Tunnel". Technology of Highway and Transport, 33(5), 2017, pp.132-135.

6. Wang, Y., Zhao, Y., Quan, Q.,Cao, Z., "Dynamic characteristics of external luminance of highway tunnel in cold area". In: IOP Conference Series: Earth and Environmental Science, Dalian, China: IOP Publishing Ltd, 2020, pp.012024.

7. Gueorgiev, V., "Optimization of Energy Consumption of Road Tunnels". In: 2020 Fifth Junior Conference on Lighting (Lighting), Ruse, Bulgaria: IEEE, 2020, pp.1-5.

8. Peña-García, A., Salata, F.,Golasi, I., "Decrease of the maximum speed in highway tunnels as a measure to foster energy savings and sustainability". Energies, 12(4), 2019, pp.685.

9. Peña-García, A.,Gómez-Lorente, D., "Installation of solar panels in the surroundings of tunnel portals: A double-targeted strategy to decrease lighting requirements and consumption". Tunnelling and underground space technology, 97(1), 2020, pp.103251.

10. López, J.,Peña-García, A., "Determination of lighting and energy demands of road tunnels using vehicle based photographs of the portal gates: An accessible and safe tool for tunnel renewal and maintenance". Tunnelling and underground space technology, 78(1), 2018, pp.8-15.

11. Blaser, P.,Dudli, H., "Tunnel lighting: Method of calculating luminance of access zone L20". Lighting Research \& Technology, 25(1), 1993, pp.25-30.

12. Pachamanov, A., Pregyov, B.,Kassev, K., "Annual use of luminaire groups for the entrance area according to the orientation of the road tunnel". In: 2019 Second Balkan Junior Conference on Lighting (Balkan Light Junior), Plovdiv, Bulgaria: IEEE, 2019, pp.1-4.

13. Doulos, L. T., Sioutis, I., Tsangrassoulis, A., Canale, L.,Faidas, K., "Revision of threshold luminance levels in tunnels aiming to minimize energy consumption at no cost: Methodology and case studies". Energies, 13(7), 2020, pp.1707.
14. Xu, J., Wu, J.,He, Y., "Discussion on Dynamic Characteristic of Road Tunnel Adaptation Luminance". Lamps and lighting, 42(1), 2018, pp.10-15.

15. Deng, M.,Zhang, F., "Method obtaining scenery luminance outside tunnel portals with in-situ testing". Journal of civil, Architectural \& Environmental Engineering, 38(3), 2016, pp.118-122.

16. Zhang, T., "Research of Determining Typical Elements' Luminance at Tunnel Portals By Radiation-Illuminance Conversion Method". Master thesis of Chongqing University, China, 2016, pp.49-77.

17. He, W.,Liang, B., "A Novel Method for Calculating Luminance in the Access Zone of a Road Tunnel based on a Digital Camera". Journal of Engineering Science and Technology Review, 14(3), 2021, pp.148 - 157.

18. Xiao, Q., "Measurement of Tunnel Luminance and Vehicle Detection Based on Video Stream and its Application". Master thesis of Guilin university of electronic technology, China, 2018, pp.16-33.

19. Liang, B.,He, W., "Slope Illuminance Prediction under a Cloudless Sky Based on the Novel Model for Global Horizontal Irradiance and Illuminance". Journal of Engineering Science and Technology Review, 14(4), 2021, pp.135-145.

20. Wu, Y., Liu, C.,Wen, S., "Calculation of Sky Luminance Distribution at Arbitrary Time Based on the CIE Sky Model". Acta Optical Sinica, 34(11), 2014, pp.27-33.

21. Perez, R., Seals, R., Ineichen, P., Stewart, R.,Menicucci, D., "A new simplified version of the Perez diffuse irradiance model for tilted surfaces". Solar Energy, 39(3), 1987, pp.221-231.

22. Perez, R., Ineichen, P., Seals, R., Michalsky, J.,Stewart, R., "Modeling daylight availability and irradiance components from direct and global irradiance". Solar Energy, 44(5), 1990, pp.271289.

23. Darula, S., Kittler, R.,Wittkopf, S. K., "Outdoor illuminance levels in the tropics and their representation in virtual sky domes". Architectural Science Review, 49(3), 2006, pp.301-313.

24. Kittler, R.,Darula , S., "Determination of sky types from global illuminance". International Journal of Lighting Research and Technology, 32(4), 2000, pp.187-193.

25. Kittler, R.,Darula, S., "Parametrization problems of the very bright cloudy sky conditions". Solar Energy, 62(2), 1998, pp.93-100.

26. Darula, S.,Kittler, R., "New trends in daylight theory based on the new ISO/CIE Sky Standard: 3. Zenith luminance formula verified by measurement data under cloudless skies". Building Research Journal, 53(1), 2005, pp.9-31.

27. Meinel, A.,Meinel, M., “Applied Solar Energy". Boston: Addison Wesley Publishing Co., America, 1976, pp.37-43.

28. Ineichen, P., Perez, R.,Seals, R., "The importance of correct albedo determination for adequately modeling energy received by tilted surfaces". Solar Energy, 39(4), 1987, pp.301-305. 